\title{
Extended middle fossa approach with anterior petrosectomy for resection of upper petroclival meningioma involving Meckel's cave: operative video and technical nuances
}

\begin{abstract}
James K. Liu, MD
Department of Neurological Surgery, Center for Skull Base and Pituitary Surgery, Neurological Institute of New Jersey, Rutgers University New Jersey Medical School, Newark, New Jersey

The surgical management of petroclival meningiomas remains a formidable challenge. These tumors are deep in the base of the skull and arise medial to the fifth cranial nerve. In this operative video, the author demonstrates the extended middle fossa approach with anterior petrosectomy to resect an upper petroclival meningioma extending into Meckel's cave with brainstem compression. This approach is very useful for accessing deep tumors located above and below the tentorium, and between the fifth and seventh cranial nerves. Access to Meckel's cave is readily achieved by opening the fibrous ring of the porous trigeminus. This video demonstrates the operative technique and surgical nuances of the skull base approach, useful anatomic landmarks of the middle fossa rhomboid for safe petrosectomy drilling, pearls for cranial nerve and neuro-otologic preservation, and exposure of Meckel's cave. A gross-total resection was achieved, and the patient was neurologically intact. In summary, the extended middle fossa approach with anterior petrosectomy is an important strategy in the armamentarium for surgical management of petroclival meningiomas.
\end{abstract}

The video can be found here: https://youtu.beljttwJIYPHC8.

KEYWORDS skull base meningioma; extended middle fossa; anterior petrosectomy; Kawase approach; petroclival meningioma

SUBMITTED May 31, 2017. ACCEPTED June 28, 2017.

INCLUDE WHEN CITING Published online October 1, 2017; DOI: http://thejns.org/doi/abs/10.3171/2017.10.FocusVid.17345.

CORRESPONDENCE James K. Liu, Department of Neurological Surgery, Rutgers University New Jersey Medical School, 90 Bergen Street, Ste. 8100 , Newark, NJ 07103. email: james.liu.md@rutgers.edu. 